Pacific Journal of Mathematics

KERNELS OF COMPOUND GAMES WITH SIMPLE 


\title{
KERNELS OF COMPOUND GAMES WITH SIMPLE COMPONENTS
}

\author{
NimRod Megiddo
}

The kernel is a solution concept for a cooperative game. It reflects symmetry properties of the characteristic function and desirability relations over the set of the players. Given $m$ games over disjoint sets of players and an $m$-person game, one defines a compound game over the union of the $m$ disjoint sets. These $m$ games are the components and the above $m$ person game is called the quotient. The quotient may be treated as a game played by representatives of the component games.

The kernel of the compound game is characterized fully. The compound kernel is, in fact, a composition of the components' kernels by means of a distinguished subset of the imputation space of the quotient game. This subset depends also on the number of veto players in each component.

An effective formula for the compound kernel is given for compound simple games. This formula enables short cuts in the computations leading to the kernel of a decomposable game. The results are applied to compound majority games and a complete description of their kernels is given.

1. Introduction. The kernel of a characteristic function game was defined by M. Davis and M. Maschler in [2] and it is related to the theory of bargaining sets. M. Maschler and B. Peleg $([4,5])$ presented many interesting properties of the kernel. The kernel reflects strength relations between players and symmetry properties of the characteristic function.

Compound simple games were defined by L. S. Shapley in [11]. In this paper we deal with compound games which are not necessarily simple, but their components are simple (see [13; p. 29]). The decomposability of games was investigated by Shapley in [15] and by the present author in [7].

This paper aims at describing the kernel of a compound game in terms of the quotient game and the kernels of the components. In fact, we introduce a subset of the imputations space of the quotient game which determines the structure of the kernel of the compound game. The kernels of the components are composed according to a formula which depends on that subset and generate the compound kernel. The formula is shown to be effective for computation and it can be simplified when the quotient game is also simple.

L. S. Shapley $([12,13,14])$ and G. Owen ([9]) proved that vonNeumann-Morgenstern solutions of the component games compose in 
a natural manner which results in a solution of the compound game. B. Peleg gave a characterization of the kernel of another kind of composition for games ([10]). The nucleolus of a compound game was characterized in [8]. The kernel of a product of simple games was characterized in [6]. This paper generalizes the results of [6] with respect to the kernel.

2. Preliminaries. A characteristic function game is a pair $\Gamma=$ $(N ; v)$, where $N$ is a nonempty finite set $(N=\{1, \cdots, n\})$ and $v$ is a real-valued function defined over the subsets of $N$. The elements of $N$ are the players and the subsets of $N$ are the coalitions.

If for every coalition $S$ either $v(S)=0$ or $v(S)=1$ then we call the game a simple game. Those coalitions that have a unit value are called winning coalitions. The set of the winning coalitions is denoted by $\mathscr{W}$ and the game is represented also by $(N ; \mathscr{W})$. We always assume $\varnothing \notin \mathscr{W}$ and $N \in \mathscr{W}$.

A game is said to be monotonic if for every pair of coalitions $S, T$.

$$
S \subset T \Longrightarrow v(S) \leqq v(T) .
$$

A 1-normalized game is a game $(N ; v)$ such that

$$
v(N)=1 \text {. }
$$

A 1-0-normalized game is a 1-normalized game $(N ; v)$ such that

$$
v(\{i\})=0 \quad(i=1, \cdots, n) .
$$

We assume that always

$$
v(\varnothing)=0
$$

A player $i \in N$ is called dummy if for every coalition $S$

$$
v(S \cup\{i\})=v(S) \text {. }
$$

Notice that if $i$ is a dummy then according to (2.4)

$$
v(\{i\})=0 \text {. }
$$

A player $i$ in a simple game $\Gamma=(N$; $\mathscr{W})$ is called veto player if $i \in S$ for every $S \in \mathscr{W}$.

A compound game is defined as follows. Let $\Gamma_{i}=\left(N_{i}\right.$; $\left.\mathscr{W}^{i}\right)$, $i=1, \cdots, m$, be $m$ simple games over disjoint sets of players. Let $\Gamma_{0}=(M ; u)$ be an $m$-player characteristic function game $(M=\{1, \cdots$, $m\})$. The compound game $\Gamma=\Gamma_{0}\left[\Gamma_{1}, \cdots, \Gamma_{m}\right]$ is defined over the set $N=N_{1} \cup \cdots \cup N_{m}$ and its characteristic function $v$ is defined by

$$
v(S)=u\left(\left\{i \in M: S \cap N_{i} \in \mathscr{W}^{i}\right\}\right) \quad(S \subset N) .
$$


$\Gamma_{0}$ is the quotient and $\Gamma_{1}, \cdots, \Gamma_{m}$ are the components. Thus, a coalition in the compound game has the value (in the quotient) of the set of those components in which it has enough players to form a winning coalition (in that game). The concept of a compound game contains as particular cases the product and the sum of simple games. The product of two simple games $\Gamma_{1}, \Gamma_{2}$ is defined by

$$
\Gamma_{1} \otimes \Gamma_{2}=B_{2}\left[\Gamma_{1}, \Gamma_{2}\right]
$$

and their sum is defined by

$$
\Gamma_{1} \oplus \Gamma_{2}=B_{2}^{*}\left[\Gamma_{1}, \Gamma_{2}\right]
$$

where $B_{2}$ and $B_{2}^{*}$ are defined by

$$
\begin{gathered}
B_{2}=(\{1,2\} ;\{1,2\}) \\
B_{2}^{*}=(\{1,2\} ;\{1\},\{2\},\{1,2\}) .
\end{gathered}
$$

An imputation in an $n$-player game $\Gamma=(N ; v)$ in an $n$-tuple of real numbers $x=\left(x_{1}, \cdots, x_{n}\right)$ such that

$$
x_{i} \geqq v(\{i\}) \quad(i=1, \cdots, n)
$$

and

$$
\sum_{i \in N} x_{i}=v(N) .
$$

The set of the imputations is denoted by $\mathscr{C}(\Gamma)$. A pseudo-imputation is an $n$-tuple of nonnegative numbers $x=\left(x_{1}, \cdots, x_{n}\right)$ that satisfies (2.13). A weak imputation is defined by (2.12) and

$$
\sum_{i \in N} x_{i} \leqq v(N) .
$$

The set of the weak imputations will be denoted by $\widetilde{\mathscr{P}}(\Gamma)$. For every coalition $S$ we denote

$$
\begin{gathered}
x(S)=\sum_{i \in S} x_{i}, \quad(x(\varnothing)=0) \\
e(S, x)=v(S)-x(S)
\end{gathered}
$$

and call $e(S, x)$ the excess of $S$ with respect to $x$. The maximum surplus of a player $i$ against another player $j$ with respect to $x$ is defined by

$$
s_{i j}(x)=\operatorname{Max}\{e(S, x): S \subset N, i \in S, j \notin S\} .
$$

The kernel (for the grand coalition) of a game $\Gamma=(N ; v)$ is defined to be the set $\mathscr{K}(\Gamma)$ of all the imputations $x \in \mathscr{P}(\Gamma)$ such that for every pair of distinct players $i, j \in N, x_{j}>v(\{j\})$ implies 


$$
s_{i j}(x) \leqq s_{j i}(x) .
$$

Equivalently, $x$ belongs to the kernel of the game if and only if for every pair of distinct players $i, j$

$$
\left[s_{i j}(x)-s_{j i}(x)\right] \cdot\left[x_{j}-v(\{j\})\right] \leqq 0 .
$$

The kernel is nonempty whenever $\mathscr{P}(\Gamma)$ is nonempty (see [2]) and for monotonic games in 1-0-normalization (2.19) may be changed to

$$
s_{i j}(x)=s_{j i}(x)
$$

(see [5; Corollary 3.9]). The pseudo-kernel ${ }^{1}$ of a game is the set of all the pseudo-imputations $x$ such that for every pair of distinct players

$$
\left[s_{i j}(x)-s_{j i}(x)\right] \cdot x_{j} \leqq 0 .
$$

Let a transformation $T$ from $E^{r}$ into $E^{r}$ be defined by

$$
T x=\frac{A x+b}{\langle c, x\rangle+\delta}
$$

where $A$ is a linear transformation of $E^{r}$ into itself, $b$ and $c$ are $r$-dimensional vectors and $\delta$ is a real number. We assume that if $c$ is the zero vector then $\delta \neq 0$. Any transformation of this type will be called a projective transformation of $E^{r}$. Note that it is not defined for $x$ in $N(T)=\{y:\langle c, y\rangle+\delta=0\}$; this set may be empty though. Convexity is preserved by projective transformations, i.e., if $T$ is a projective transformation of $E^{r}$ and if $P \subset E^{r}$ is such that $T$ is defined for every $x$ in conv $P$ then

$$
T(\operatorname{conv} P)=\operatorname{conv} T(P) \text {. }
$$

An $s$-variable transformation $T$ from $\mathbf{X}_{i=1}^{s} E_{i}^{r}\left(E_{i}^{r}=E^{r}, i=1, \cdots, s\right)$ into $E^{r}$ is called a multi-projective transformation if for every $i, i=1$, $\cdots, s$, and fixed $x^{1}, \cdots, x^{i-1}, x^{i-1}, \cdots, x^{s}$ in $E^{r}$ the transformation $T_{i}: E^{r} \rightarrow E^{r}$ defined by

$$
T_{i}(x)=T\left(x^{1}, \cdots, x^{i-1}, x, x^{i-1}, \cdots, x^{s}\right)
$$

is a projective transformation of $E^{r}$. If $P_{i} \subset E_{i}^{r}, i=1, \cdots, s$, are sets such that the multi-projective transformation $T$ is defined for every $\left(x^{1}, \cdots, x^{s}\right) \in \operatorname{conv} P_{1} \times \cdots \times \operatorname{conv} P_{s}$ then

$$
T\left(\operatorname{conv} P_{1} \times \cdots \times \operatorname{conv} P_{s}\right)=\operatorname{conv} T\left(P_{1} \times \cdots \times P_{s}\right) .
$$

3. Basic lemmas. We assume that for every player $i \in N$ in $(N ; v)$

$$
v(\{i\})=0 \text {. }
$$

${ }^{1}$ All the statements in this paper hold for the pseudo-kernel of a game which is not necessarily 1-0-normalized. We do not use explicitly the normalization assumption. The reader is referred to [5; p. 573] for a clearification of this point. 
Notice that if $i \in N_{k}$ is a player who do not satisfy (3.1) in the compound game then $\{i\} \in \mathscr{W}^{k}$ and therefore either the kernel of $\Gamma_{k}$ is empty, or it consists of a unique point where $i$ gets a unit payoff and the other players in $\Gamma_{k}$ get zero. Our compound games are assumed to be monotonic and dummy-free. We also assume that for every component game $\Gamma_{k}=\left(N_{k} ; \mathscr{W}^{k}\right), k=1, \cdots, m, N_{k} \in \mathscr{W}^{k}$ and $\varnothing \notin \mathscr{W}^{k}$. It is left to the reader to verify that our assumptions imply that the component games are also monotonic and dummy-free.

Lemma 3.1. Let $\mathscr{K}$ be the kernel of a simple game $\Gamma=(N$; $\mathscr{W})$. Denote

$$
\mu(x)=\min \{x(S): S \in \mathscr{W}\} \quad(x \in \mathscr{X}(\Gamma)) .
$$

There exist convex polyhedra $K_{1}, \cdots, K_{r}$ such that $\mathscr{K}=\bigcup_{i=1}^{r} K_{i}$ and such that $\mu(x)$ is linear in each $K_{i}, i=1, \cdots, r$.

Proof. $\mathscr{K}(\Gamma)$ is a finite union of convex polyhedra (see $[1 ; \S 3])$. The required polyhedra are the nonempty intersections of the form $P_{i} \cap H_{S}$ where

$$
H_{S}=\left\{x \in E^{n}: x(S) \leqq x(T) \text { for every } T \in \mathscr{W}\right\} \quad(S \in \mathscr{W})
$$

and $P_{i}$ are the polyhedra assured by [1].

For every player $i$ and $x \in \widetilde{\mathscr{P}}(\Gamma)$ let us denote

$$
\begin{gathered}
g_{i}(x)=\operatorname{Max}\{e(S, x): i \in S \subset N\} \\
h_{i}(x)=\operatorname{Max}\{e(S, x): i \notin S \subset N\} .
\end{gathered}
$$

LEMMA 3.2. Let $\Gamma=(N ; v)$ be a monotonic game satisfying (3.1). If $x \in \mathscr{K}(\Gamma)$ then for every $i \in N$

$$
g_{i}(x)=h_{i}(x)
$$

(and therefore $g_{i}(x)=s(x) \equiv \operatorname{Max}\{e(S, x): S \subset N\}-$ see (3.4)-(3.5)).

Proof. Since for every pair of distinct players $i, j s_{i j}(x)=s_{j i}(x)$ $(x \in \mathscr{K}(\Gamma))$, it follows that

$$
\begin{aligned}
g_{i}(x) & =\operatorname{Max}\{e(S, x): i \in S \subset N\} \\
& =\operatorname{Max}[\operatorname{Max}\{e(S, x): i \in S \varsubsetneqq N\}, 0] \\
& =\operatorname{Max}\left[\operatorname{Max}\left\{s_{i j}(x): j \in N, j \neq i\right\}, 0\right] \\
& =\operatorname{Max}\left[\operatorname{Max}\left\{s_{j i}(x): j \in N, j \neq i\right\}, 0\right] \\
& =\operatorname{Max}[\operatorname{Max} e(S, x): i \notin S, \varnothing \neq S \subset N\}, 0] \\
& =\operatorname{Max}\{e(S, x): i \notin S \subset N\} \\
& =h_{i}(x) .
\end{aligned}
$$


If $i, j$ are two distinct players in $(N ; v)$ we denote

$$
\mathscr{T}_{i j}=\{S \subset N: i \in S, j \notin S\}
$$

and if the game is simple, $(N ; \mathscr{W})$, denote

$$
\mathscr{W}_{i j}=\mathscr{W} \cap \mathscr{T}_{i j} \text {. }
$$

Also,

$$
\begin{aligned}
& a_{i j}(x)=\operatorname{Max}\{e(S, x): i \notin S, j \notin S, \varnothing \neq S \subset N\} \quad(x \in \widetilde{\mathscr{C}}(\Gamma)) \\
& b_{i j}(x)=\operatorname{Max}\{e(S, x): i \in S, j \in S, S \subset N\} \quad(x \in \widetilde{\mathscr{X}}(\Gamma)) .
\end{aligned}
$$

Given an imputation $x \in \mathscr{P}(\Gamma)$ in a compound game $\Gamma=(N ; v)$ we denote by $\mu$ a weak imputation in $\Gamma_{0}$ (see (2.7))

$$
\mu \equiv \mu[x]=\left(\mu_{1}[x], \cdots, \mu_{m}[x]\right)
$$

where

$$
\mu_{k}[x]=\min \left\{x(S): S \in \mathscr{W}^{k}\right\} \quad(k=1, \cdots, m) .
$$

We will write $e^{k}(S, x), s^{k}(x)$ (see Lemma 3.2), $\mathscr{T}_{i j}^{k}$, $\mathscr{W}_{i j}^{k}, s_{i j}^{k}(x), g_{i}^{k}(x)$, $h_{i}^{k}(x), a_{i j}^{k}(x), b_{i j}^{k}(x)$, where these expressions refer to the game $\Gamma_{k}, k=0$, $1, \cdots, m$. Note that

$$
s^{k}(x)=1-\mu_{k}[x] .
$$

Lemma 3.4. Let $\Gamma=(N ; v)$ be a compound game, let $x \in \mathscr{X}(\Gamma)$ and let $i, j \in N_{k}$ be two distinct players belonging to the same component game $\Gamma_{k}, k=1, \cdots, m$.

(i) If $j$ is not a veto player in $\Gamma_{k}$ then

$$
s_{i j}(x)=\operatorname{Max}\left[g_{k}^{0}(\mu)+s_{i j}^{k}(x)-s^{k}(x), h_{k}^{0}(\mu)-x_{i}\right] .
$$

(ii) If $j$ is a veto player in $\Gamma_{k}$ then

$$
s_{i j}(x)=h_{k}^{0}(\mu)-x_{i} .
$$

Proof. (i) If $j$ is not a veto players in $\Gamma_{k}$ then there exists a coalition $S \in \mathscr{W}^{k}$ such that $j \notin S$. Thus, $S \cup\{i\} \in \mathscr{W}_{i j}^{k}$. Considering the compound game, we find that

$$
\begin{aligned}
s_{i j}(x)= & \operatorname{Max}\left\{e(S, x): S \in \mathscr{T}_{i j}\right\} \\
= & \operatorname{Max}\left[\operatorname{Max}\left\{e(S, x): S \in \mathscr{T}_{i j}, S \cap N_{k} \in \mathscr{W}^{k}\right\},\right. \\
& \left.\quad \operatorname{Max}\left\{e(S, x): S \in \mathscr{T}_{i j}, S \cap N_{k} \notin \mathscr{W}^{k}\right\}\right] \\
= & \operatorname{Max}[\operatorname{Max}\{u(T)-\mu(T \backslash\{k\}): k \in T \subset M\}-\min \{x(S): S \in \\
& \left.\quad \mathscr{W}_{i j}^{k}\right\}, \\
& \left.\operatorname{Max}\{u(T)-\mu(T): k \notin T \subset M\}-x_{i}\right] \\
= & \operatorname{Max}\left[\operatorname{Max}\left\{e^{0}(T, \mu): k \in T \subset M\right\}+\mu_{k}-\min \left\{x(S): S \in \mathscr{W}_{i j}^{k}\right\},\right. \\
& \left.\quad \operatorname{Max}\left\{e^{0}(T, \mu): k \notin T \subset M\right\}-x_{i}\right] \\
= & \operatorname{Max}\left[g_{k}^{0}(\mu)+s_{i j}^{k}(x)-s^{k}(x), h_{k}^{0}(\mu)-x_{i}\right] .
\end{aligned}
$$


(ii) If $j$ is a veto player in $\Gamma_{k}$ then $\mathscr{W}_{i j}^{k}=\varnothing$ and

$$
\begin{aligned}
s_{i j}(x) & =\operatorname{Max}\left\{e(S, x): S \in \mathscr{T}_{i j}, S \cap N_{k} \notin \mathscr{W}^{k}\right\} \\
& =\operatorname{Max}\left\{e^{0}(T, \mu): k \notin T \subset M\right\}-x_{i} \\
& =h_{k}^{\circ}(\mu)-x_{i} .
\end{aligned}
$$

LeMma 3.5. Let $\Gamma=(N ; v)$ be a compound game, let $x \in \mathscr{X}(\Gamma)$ and let $i \in N_{k}, j \in N_{l}$ be two players belonging to distinct component games $\Gamma_{k}, \Gamma_{l}, 1 \leqq k<l \leqq m$.

(i) If $j$ is not a veto player in $\Gamma_{l}$ then

$$
\begin{gathered}
s_{i j}(x)=\operatorname{Max}\left[s_{k l}^{0}(\mu)+g_{i}^{k}(x)-s^{k}(x), a_{k l}^{0}(\mu)-x_{i},\right. \\
b_{k l}^{0}(\mu)+g_{i}^{k}(x)-s^{k}(x)+h_{j}^{l}(x)-s^{l}(x), \\
\left.s_{l k}^{0}(\mu)+h_{j}^{l}(x)-s^{l}(x)-x_{i}\right] .
\end{gathered}
$$

(ii) If $j$ is a veto player in $\Gamma_{l}$ then

$$
s_{i j}(x)=\operatorname{Max}\left[s_{k l}^{0}(\mu)+g_{i}^{k}(x)-s^{k}(x), a_{k l}^{0 l}(\mu)-x_{i}\right] .
$$

The proof is similar to that of Lemma 3.4.

Lemma 3.6. Let $\Gamma=(N ; v)$ be a compound game and let $x \in \mathscr{K}(\Gamma)$. For every $k, k=1, \cdots, m$,

$$
\mu_{k}[x]=0 \Longleftrightarrow x\left(N_{k}\right)=0 \text {. }
$$

Proof. Assume that $\mu_{k}[x]=0$ and let $S_{0} \in \mathscr{W}^{k}$ such that $x\left(S_{0}\right)=0$. Clearly, for all $i, j$ such that $S_{0} \in \mathscr{T}_{i j} s_{i j}^{k}(x)=1$. Hence

$$
s^{k}(x)=1 \text {. }
$$

Assume that $x\left(N_{k}\right)>0$ and let $j \in N_{k}$ such that $x_{j}>0 . \quad j \notin S_{0}$ and therefore $j$ is not a veto player. Let $i \in S_{0}$. According to Lemma 3.4,

$$
\begin{aligned}
s_{i j}(x) & =\operatorname{Max}\left[g_{k}^{0}(\mu)+s_{i j}^{k}(x)-s^{k}(x), h_{k}^{0}(\mu)-x_{i}\right] \\
& =\operatorname{Max}\left[g_{k}^{0}(\mu), h_{k}^{0}(\mu)\right] \\
& =s^{0}(\mu) .
\end{aligned}
$$

On the other hand,

$$
\begin{aligned}
s_{j_{i}}(x) & \leqq g_{j}(x) \\
& =\operatorname{Max}\left[g_{k}^{0}(\mu)+\mu_{k}-\min \{x(S): j \in S \in \mathscr{W}\}, h_{k}^{0}(\mu)-x_{j}\right] \\
& \leqq \operatorname{Max}\left[g_{k}^{0}(\mu)-x_{j}, h_{k}^{0}(\mu)-x_{j}\right] \\
& =s^{0}(\mu)-x_{j} \\
& <s^{0}(\mu) .
\end{aligned}
$$

It follows that $s_{i j}(x)>s_{j i}(x)$ in contradiction to our assumption that $x \in \mathscr{K}(\Gamma)$. The other direction of (3.21) is immediate. 
LEmma 3.7. Let $\Gamma=(N ; v)$ be a compound game and let $x \in \mathscr{K}(\Gamma) . \quad$ For every $k, k=1, \cdots, m$,

$$
g_{k}^{0}(\mu)=s(x) \text {. }
$$

Proof. If $\mu_{k}=0$ then for every $T \subset M$

It follows that

$$
\begin{aligned}
e^{0}(T \cup\{k\}, \mu) & =u(T \cup\{k\})-\mu(T \cup\{k\}) \\
& =u(T \cup\{k\})-\mu(T) \\
& \geqq u(T)-\mu(T) \\
& =e^{0}(T, \mu) .
\end{aligned}
$$

$$
g_{k}^{0}(\mu)=s^{0}(\mu)=s(x) .
$$

Suppose $\mu_{k}>0$ and let $i \in N_{k}$ such that $x_{i}>0$ and

$$
g_{i}^{k}(x)=s^{k}(x) \text {. }
$$

Thus,

$$
g_{i}(x)=\operatorname{Max}\left[g_{k}^{0}(\mu), h_{k}^{0}(\mu)-x_{i}\right] .
$$

If $i$ is not a veto player in $\Gamma_{k}$ then

$$
h_{i}(x)=\operatorname{Max}\left[g_{k}^{0}(\mu)+h_{i}^{k}(x)-s^{k}(x), h_{k}^{0}(\mu)\right] .
$$

In this case it follows from Lemma 3.2 and (3.29)-(3.30) that

$$
g_{k}^{0}(\mu) \geqq h_{k}^{0}(\mu)
$$

and this is equivalent to (3.25). If $i$ is a veto player then

$$
h_{i}(x)=h_{k}^{0}(\mu) \text {. }
$$

In this case (3.31) follows from Lemma 3.2, (3.29), and (3.32).

REMARK 3.8. If $i$ is a veto player in $\Gamma_{k}$ then it follows from Lemma 3.2, (3.29), and (3.32) that

$$
g_{k}^{0}(\mu)=h_{k}^{0}(\mu) .
$$

4. On the kernel of a component game. The barycentric projection of an imputation $x \in \mathscr{X}(\Gamma)$ on a coalition $S$ such that $x(S)>0$ will be denoted (see [13; p. 6]) by $B_{S} x$ and defined to be an $|S|$-tuple $B_{S} x=\left[\left(B_{S} x\right)_{i}\right]_{i \in S}$ where for every $i \in S$

$$
\left(B_{S} x\right)_{i}=\frac{x_{i}}{x(S)} .
$$

Notice that if $\Gamma$ is a compound game and $\Gamma_{k}=\left(N_{k} ; \mathbb{W}^{k}\right)$ is a component game of $\Gamma$ then $B_{N_{k}} x$ is a pseudo-imputation in $\Gamma_{k}$ or even an imputation if (3.1) is satisfied in $\Gamma_{k}$. 
Theorem 4.1. Let $\Gamma=(N ; v)$ be a compound game and let $x \in \mathscr{P}(\Gamma)$ be an imputation such that for every $k, k=1, \cdots, m$,

$$
g_{k}^{0}(\mu)=s(x) \text {. }
$$

Under these conditions, if $x\left(N_{k}\right)>0$ and $\Gamma_{k}$ is a game with veto players then

$$
B_{N_{k}} x \in \mathscr{K}\left(\Gamma_{k}\right)
$$

if and only if for every pair of distinct players $i, j \in N_{k}$

$$
\left[s_{i j}(x)-s_{j i}(x)\right] \cdot x_{j} \leqq 0 .
$$

Proof. The kernel of a simple game with veto players consists of a unique point in which the veto players share equally while the others get zero ([5; Theorem 4.1]).

(a) Suppose $B_{N_{k}} x \in \mathscr{K}\left(\Gamma_{k}\right)$ and let $i, j$ be two distinct players in $\Gamma_{k}$. If both of them are veto players then (see Lemma 3.4)

$$
\begin{aligned}
& s_{i j}(x)=h_{k}^{0}(\mu)-x_{i} \\
& s_{j i}(x)=h_{k}^{0}(\mu)-x_{j}
\end{aligned}
$$

and since $x_{i}=x_{j}$ it follows that $s_{i j}(x)=s_{j i}(x)$. If $j$ is a veto player but $i$ is not, then (notice that $s_{i j}^{k}(x)=s^{k}(x)$ since all the winning coalitions in $\Gamma_{k}$ have the same excess $1-x\left(N_{k}\right)$ )

$$
s_{i j}(x)=\operatorname{Max}\left[g_{k}^{0}(\mu), h_{k}^{0}(\mu)-x_{j}\right] .
$$

According to (4.5) (it holds when $i$ is not a veto player) and the fact that $x_{i}=0$, it follows that

$$
s_{i j}(x)=h_{k}^{0}(\mu) .
$$

Considering (4.2) it follows that $s_{i j}(x) \leqq s_{j i}(x)$. If $j$ is not a veto player (4.4) follows from the fact that $x_{j}=0$.

(b) Suppose (4.4) is true for every pair of distinct players $i, j$. (4.5)-(4.6) hold for every pair of veto players. According to (4.4) $x_{i}=x_{j}$. Suppose, per absurdum, that there is $j \in N_{k}$, who is not a veto player, such that $x_{j}>0$. Let $i$ be a veto player in $\Gamma_{k}$. (4.4) implies $s_{i j}(x) \leqq s_{j i}(x)$. Thus, according to Lemma 3.4 and (4.2)

$$
\begin{aligned}
s^{0}(\mu)+s_{i j}^{k}(x)-s^{k}(x) & \leqq s_{i j}(x) \leqq s_{j i}(x) \\
& =h_{k}^{0}(\mu)-x_{j}<s^{0}(\mu)
\end{aligned}
$$

and hence

$$
s_{i j}^{k}(x)<s^{k}(x)
$$


This means that $j$ belongs to every winning coalition having a maximal excess ((4.10) holds for each veto player $i)$. Thus, a coalition $S$ that has a maximal excess contains all the veto players and all the other players of positive payoff. Therefore,

$$
s^{k}(x)=1-x\left(N_{k}\right) .
$$

It follows that all the winning coalitions have the same excess in contradiction to (4.10). This contradiction proves that each player $j$ who is not a veto player gets zero and therefore $B_{N_{k}} x$ belongs to the kernel.

THEOREM 4.2. Let $\Gamma=(N ; v)$ be a compound game and let $x \in \mathscr{X}(\Gamma)$ be an imputation satisfying (4.2). Under these conditions, if $\Gamma_{k}$ is free of veto players and $x\left(N_{k}\right)>0$ then

$$
B_{N_{k}} x \in \mathscr{K}\left(\Gamma_{k}\right)
$$

if and only if for every pair of distinct players $i, j \in N_{k}$

$$
s_{i j}(x)=s_{j i}(x) \text {. }
$$

Proof. Let $i, j$ be any two distinct players in $\Gamma_{k}$ and denote

$$
\begin{gathered}
\hat{x}=B_{N_{k}} x \\
\Delta=g_{k}^{0}(\mu)-h_{k}^{0}(\mu) \\
\hat{\jmath}=\frac{\Delta}{x\left(N_{k}\right)} .
\end{gathered}
$$

It follows from (4.2) (note that $s(x)=s^{0}(\mu)$ ) that

$$
\Delta, \hat{\Delta} \geqq 0 \text {. }
$$

Using Lemma 3.4 we find that

$$
\begin{aligned}
s_{i j}(x) & =\operatorname{Max}\left[g_{k}^{0}(\mu)+s_{i j}^{k}(x)-s^{k}(x), h_{k}^{0}(\mu)-x_{i}\right] \\
& =g_{k}^{0}(\mu)-s^{k}(x)+\operatorname{Max}\left[s_{i j}^{k}(x), s^{k}(x)-\Delta-x_{i}\right]
\end{aligned}
$$

and it follows that

$$
\begin{aligned}
s_{i j}(x)=s_{j i}(x) \Longleftrightarrow & \operatorname{Max}\left[s_{i j}^{k}(\hat{x}), s^{k}(\hat{x})-\hat{\Delta}-\hat{x}_{i}\right] \\
& =\operatorname{Max}\left[s_{j i}^{k}(\hat{x}), s^{k}(\hat{x})-\hat{\Delta}-\hat{x}_{j}\right] .
\end{aligned}
$$

Suppose (4.13) is satisfied for every pair of distinct players $i, j \in N_{k}$. We will show that for every $j \in N_{k}$ ( $j$ is not a veto player)

$$
h_{j}^{k}(\widehat{x}) \geqq g_{j}^{k}(\hat{x}) \text {. }
$$


Indeed, if $h_{j}^{k}(\hat{x})<g_{j}^{k}(\hat{x})$ then there exists $S_{0}$ such that $j \in S_{0}$ and

$$
h_{j}^{k}(\hat{x})<e^{k}\left(S_{0}, \hat{x}\right)=s^{k}(\hat{x}) .
$$

Let $i \in N_{k} \backslash S_{0}\left((4.21)\right.$ implies $e^{k}\left(S_{0}, \hat{x}\right)>0$ and therefore $\left.S_{0} \neq N_{k}\right)$. Clearly,

$$
s_{i j}^{k}(\hat{x})<s^{k}(\hat{x})=s_{i j}^{k}(x) .
$$

Since

(4.23) $\operatorname{Max}\left[s_{i j}^{k}(\hat{x}), s^{k}(\hat{x})-\hat{\Delta}-\widehat{x}_{i}\right]=\operatorname{Max}\left[s_{j i}^{k}(\hat{x}), s^{k}(\hat{x})-\hat{\Delta}-\hat{x}_{j}\right]$

it follows that

$$
\widehat{x}_{i}=\hat{\Delta}=0 .
$$

The last equality is true for every $i \notin S_{0}$ so that $x\left(S_{0}\right)=1$ in contradiction to (4.21). Thus, (4.20) is proved. Let $S_{0} \subset N_{k}$ be a coalition such that $j \notin S_{0}$ and

$$
e^{k}\left(S_{0}, \hat{x}\right)=s^{k}(\hat{x}) .
$$

Since for every $i \in N_{k}(i \neq j)$

$$
e^{k}\left(S_{0} \cup\{i\}, \widehat{x}\right) \geqq e^{k}\left(S_{0}, \widehat{x}\right)-\widehat{x}_{i}=s^{k}(\widehat{x})-\widehat{x}_{i}
$$

it follows that

$$
s_{i j}^{k}(\hat{x}) \geqq s^{k}(\widehat{x})-\widehat{x}_{i}
$$

Analogously,

$$
s_{j:}^{h}(\hat{x}) \geqq s^{k}(\hat{x})-\hat{x}_{j} .
$$

It follows from (4.17), (4.19), and (4.27)-(4.28) that

$$
s_{i j}^{k}(\widehat{x})=s_{j i}^{k}(\widehat{x})
$$

and hence $x \in \mathscr{K}\left(\Gamma_{k}\right)$. On the other hand, if $\hat{x} \in \mathscr{K}\left(\Gamma_{k}\right)$ then (4.29) is satisfied by every pair of distinct players $i, j \in N_{k}$. Lemma 3.2 implies $h_{j}^{h}(\hat{x})=s^{k}(\hat{x})$. Hence, there is $S_{0} \subset N_{k}$ such that $j \notin S_{0}$ and $\mathbf{s}^{k}(\hat{x})=$ $e^{k}\left(S_{0}, \hat{x}\right)=h_{j}^{k}(\hat{x})$. Thus,

$$
s_{i j}^{k}(\hat{x}) \geqq e^{k}\left(S_{0} \cup\{i\}, \hat{x}\right) \geqq e^{k}\left(S_{0}, \hat{x}\right)-\hat{x}_{i}=s^{k}(\hat{x})-\hat{x}_{i} .
$$

In a similar way we can show that (4.28) holds. (4.13) follows from (4.17), (4.19), (4.28), and (4.30).

5. The dependence on the quotient game. In the preceding section we proved that the barycentric projection of a point in the compound kernel on any component must belong to the kernel of that component (or to the pseudo-kernel if (3.1) is not satisfied in the com- 
ponent game). Moreover, if the barycentric projection of an imputation in the compound game is in the kernel of the component then the imputation must satisfy the kernel condition ((2.21)) for every pair of distinct players in that component. To complete the characterization of the kernel of the compound game we have to show how the components' kernels should be composed in order to obtain the compound kernel.

The compound kernel depends on the quotient game by means of a subset of its imputations space which is defined as follows.

DeFinition 5.1. Let $\Gamma=(M ; u)$ be a monotonic m-player game. Let $w=\left(w_{1}, \cdots, w_{m}\right)$ be an m-tuple of nonnegative numbers. The [weak] w-equalizing set $\left[\widetilde{\mathscr{P}}^{w}(\Gamma)\right] \mathscr{P}^{w}(\Gamma)$ of $\Gamma$ is defined to be the set of all the [weak] imputations $[y \in \widetilde{\mathscr{P}}(\Gamma)] y \in \mathscr{X}(\Gamma)$ that satisfy the following three conditions:

(i) For each $i, i=1, \cdots, m$,

$$
g_{i}(y)=s(y) \text {. }
$$

(ii) For every pair of distinct players $i, j \in M$, if $w_{i}=0$ and $w_{j}>0$ then

$$
s_{i j}(y)=s(y) .
$$

(iii) For every pair of distinct players $i, j \in M$, if both $w_{i}>0$ and $w_{j}>0$ then

$$
\operatorname{Max}\left[s_{i j}(y), a_{i j}(y)-\frac{y_{i}}{w_{i}}\right]=\operatorname{Max}\left[s_{j i}(y), a_{i j}(y)-\frac{y_{j}}{w_{j}}\right] .
$$

REMARK 5.2. The $w$-equalizing set for a monotonic game $\Gamma=(N ; v)$ satisfying (3.1) is a generalization of the kernel. In fact

$$
\mathscr{P}^{(1, \ldots, 1)}(\Gamma)=\mathscr{K}(\Gamma) \text {. }
$$

REMARK 5.3. $\mathscr{P}^{w}(\Gamma)\left[\widetilde{\mathscr{P}}^{w}(\Gamma)\right]$ is a finite union of convex polytopes. The number of linear inequalities which determine the $w$-equalizing set is of the same order of magnitude of that number in the kernel. When most of the $w_{i}$-s are zeroes this number is smaller than the respective number in the kernel. The computation of $\mathscr{P}^{w}(\Gamma)$ can be carried out according to [1]. We conjecture that an algorithm based on the "profile" idea can be built for $\mathscr{P}^{w}(\Gamma)$ (see $[3,4]$ ).

The $w$-equalizing set of a simple game is sufficient for determining the weak $w$-equalizing set of that game:

LEMMA 5.4. If $\Gamma=(M ; \mathscr{C})$ is a monotonic simple game without 
veto players then

$$
\widetilde{\mathscr{P}}^{w}(\Gamma)=\left\{\alpha x: x \in \mathscr{P}^{w}(\Gamma), 0 \leqq \alpha \leqq 1\right\}
$$

Proof. For every pair of distinct players $i, j \in M \mathscr{U}_{i j} \neq \varnothing$. Hence for every $x \in \tilde{\mathscr{X}}(\Gamma)$

$$
s_{i j}(x)=\operatorname{Max}\left\{e(S, x): S \in \mathscr{U}_{i j}\right\}=1-\min \left\{x(S): S \in \mathscr{U}_{i j}\right\} .
$$

Similarly,

$$
\begin{gathered}
g_{i}(x)=1-\min \{x(S): i \in S \in \mathscr{U}\} \\
s(x)=1-\min \{x(S): S \in \mathscr{U}\} .
\end{gathered}
$$

Also, if there is $S \in \mathscr{U}$ such that $i, j \notin S$ then

$$
a_{i j}(x)=1-\min \{x(S): i, j \notin S \in \mathscr{Q}\}
$$

and otherwise

$$
a_{i j}(x)=s_{i j}(x) \text {. }
$$

An imputation $x \in \mathscr{X}(\Gamma)$ satisfies the conditions of Definition 5.1 if and only if every multiplication of $x$ by $\alpha$ satisfies them. This proves (5.5).

ExAMPLE 5.5. Let $M_{3}$ denote the 3-player majority game ${ }^{2}$. The $(0,0,0)$-equalizing set for $M_{3}$ and $\mathscr{P}^{(1,0,0)}\left(M_{3}\right)$ are as illustrated.
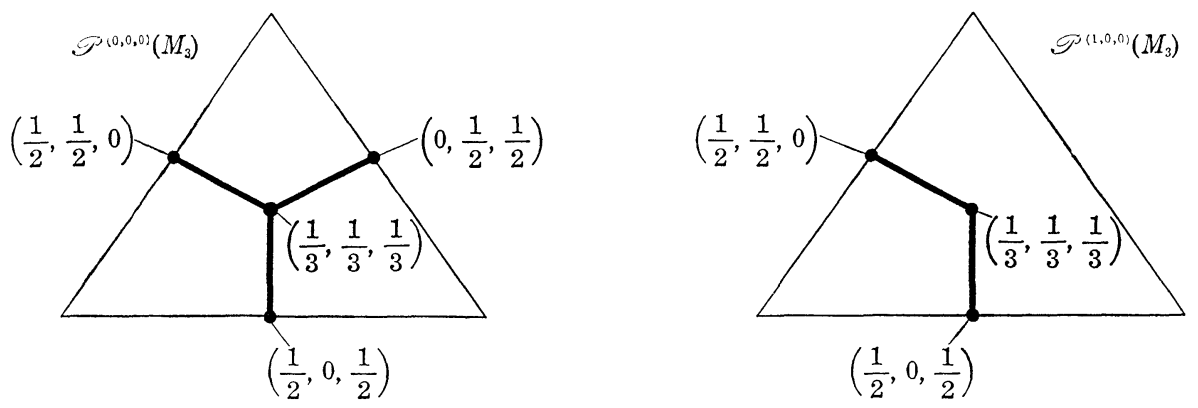

If $w_{1}, w_{2}, w_{3}>0$ then $\mathscr{P}^{w}\left(M_{3}\right)=\mathscr{K}^{2}\left(M_{3}\right)=\{(1 / 3,1 / 3,1 / 3)\}$.

The $w$-equalizing set will be now used to characterize the dependence of the compound kernel of the quotient game.

Lemma 5.6. Let $\Gamma=(N ; v)$ be a monotonic compound game satisfying (3.1). Let $x^{k} \in \mathscr{K}\left(\Gamma_{k}\right)$ and let $\alpha_{k}, k=1, \cdots, m$, be non-

${ }^{2} M_{3}$ is a 3 -player simple game in which a coalition wins if and only if it consists of at least two players.

${ }^{3}$ If (3.1) is not satisfied by the compound game or by a component then our claims remain correct provided the "kernel" is replaced by the "pseudo-kernel". 
negative numbers such that $\sum_{k=1}^{m} \alpha k=u(M)=v(N)$. Let $x^{k^{*}} \in \mathscr{P}(\Gamma)$ where $x_{i}^{k^{*}}=x_{i}^{k}$ for $i \in N_{k}$ and $x_{i}^{k^{*}}=0$ otherwise. Let $w_{k}$ denote the number of veto players in $\Gamma_{k}$. Let $x=\sum_{k=1}^{m} \alpha_{k} x^{k^{*}}$. Under these conditions

$$
x \in \mathscr{K}(\Gamma) \Longleftarrow \mu[x] \in \mathscr{\mathscr { P }}^{w}\left(\Gamma_{0}\right) .
$$

Proof. According to Lemma 3.2, for each player $i \in N_{k}(k=1$, $\cdots, m)$

$$
g_{i}^{k}(x)=h_{i}^{k}(x)=s^{k}(x) .
$$

(a) If $x \in \mathscr{K}(\Gamma)$ then Lemma 3.7 implies condition (i) (see Definition 5.1).

(b) We prove the necessity of condition (ii). Assume that $w_{k}=0$ and $w_{l}>0,1 \leqq k<l \leqq m$. Let $i \in N_{k}$ and let $j \in N_{l}$ be a veto player in $\Gamma_{l}$. Since $x^{k} \in \mathscr{K}\left(\Gamma_{k}\right)$ and $x^{l} \in \mathscr{K}\left(\Gamma_{l}\right)$, it follows from (5.12) and Lemma 3.5 that

$$
s_{i j}(x)=\operatorname{Max}\left[s_{k l}^{0}(\mu), a_{k l}^{0}(\mu)-x_{i}\right]
$$

and

$$
\begin{aligned}
s_{j i}(x)= & \operatorname{Max}\left[s_{l k}^{0}(\mu)+g_{j}^{l}(x)-s^{l}(x), a_{k l}^{0}(\mu)-x_{j},\right. \\
& b_{k l}^{0}(\mu)+g_{j}^{l}(x)-s^{l}(x)+h_{i}^{k}(x)-s^{k}(x), \\
& \left.s_{k_{l}}^{0}(\mu)+h_{i}^{k}(x)-s^{k}(x)-x_{j}\right] \\
= & \operatorname{Max}\left[s_{l k}^{0}(\mu), b_{k l}^{0}(\mu), a_{k l}^{0}(\mu)-x_{j}, s_{k l}^{0}(\mu)-x_{j}\right] \\
= & \operatorname{Max}\left[g_{l}^{0}(\mu), h_{l}^{0}(\mu)-x_{j}\right] .
\end{aligned}
$$

Note that (5.13)-(5.14) hold even if $x \notin \mathscr{K}(\Gamma)$ and (5.14) is independent of $j$ being a veto player. If $x \in \mathscr{K}(\Gamma)$ it follows from condition (i) (we have proved its necessity) and (5.14) that

$$
s_{j i}(x)=s^{0}(\mu) \text {. }
$$

Suppose $x\left(N_{k}\right)=0$. For every $T \subset M$

$$
e^{0}(T \cup\{k\}, \mu) \geqq e^{0}(T, \mu)
$$

(see (3.26)). Thus, taking the maximum over the coalitions $T$ such that $k, l \notin T$,

$$
s_{k l}^{0}(\mu) \geqq a_{k l}^{0}(\mu) .
$$

It follows from (5.13) and (5.17) that

$$
s_{i j}(x)=s_{k l}^{0}(\mu) \text {. }
$$

Since $s_{i j}(x)=s_{j i}(x)$ condition (ii) follows from (5.15) and (5.18). Assume 
$x\left(N_{k}\right)>0$. Choose $i \in N_{k}$ so that $x_{i}>0$. Thus,

$$
s^{0}(\mu) \geqq a_{k l}^{0}(\mu)>a_{k l}^{0}(\mu)-x_{i} .
$$

Since $s_{i j}(x)=s_{j i}(x)$ it follows from (5.13) and (5.15) that

$$
s^{0}(\mu)=\operatorname{Max}\left[s_{k l}^{0}(\mu), a_{k l}^{0}(\mu)-x_{i}\right]
$$

and condition (ii) follows from (5.19)-(5.20).

(c) We prove the necessity of condition (iii). Assume $w_{k}, w_{l}>0$ $(1 \leqq k<l \leqq m)$ and let $i \in N_{k}$ and $j \in N_{l}$ be veto players in their component games. According to [5; Theorem 4.1]

$$
x_{i}=\frac{\mu_{k}}{w_{k}} ; \quad x_{j}=\frac{\mu_{l}}{w_{l}} .
$$

Lemma 3.5 and (5.12) imply

$$
s_{i j}(x)=\operatorname{Max}\left[s_{k l}^{0}(\mu), a_{k l}^{0}(\mu)-\frac{\mu_{k}}{w_{k}}\right]
$$

and, symmetrically,

$$
s_{j i}(x)=\operatorname{Max}\left[s_{l k}^{\cap}(\mu), a_{k l}^{0}(\mu)-\frac{\mu_{l}}{w_{l}}\right] .
$$

The last two equalities are independent of $x$ belonging to the kernel. If $x \in \mathscr{K}(\Gamma)$ then $s_{i j}(x)=s_{j i}(x)$ and condition (iii) follows from (5.22)(5.23).

(d) Assume that $\mu[x] \in \widetilde{\mathscr{P}}^{w}\left(\Gamma_{0}\right)$ and let us prove that $x \in \mathscr{K}(\Gamma)$. Condition (i), together with Theorems 4.1-4.2, imply for every pair of distinct players $i, j \in N_{k}(k=1, \cdots, m)$

$$
\left[s_{i j}(x)-s_{j i}(x)\right] \cdot x_{j} \leqq 0 .
$$

Let $i \in N_{k}$ and $j \in N_{l}(1 \leqq k<l \leqq m)$. If $i$ and $j$ are veto players in their components then (5.22)-(5.23) hold and condition (iii) implies $s_{i j}(x)=s_{j i}(x)$. If $j$ is a veto player and $i$ is not a veto player then (5.13)-(5.14) hold and conditions (i) and (ii) imply $s_{i j}(x)=s_{j i}(x)=s^{0}(\mu)$. If both $i$ and $j$ are not veto players then (5.14) and the symmetric equality,

$$
s_{i j}(x)=\operatorname{Max}\left[g_{k}^{0}(\mu), h_{k}^{0}(\mu)-x_{i}\right],
$$

imply, according to condition (i), that $s_{i j}(x)=s_{j i}(x)=s^{0}(\mu)$. Thus, (5.24) holds for all the pairs of distinct players $i, j \in N$. Hence $x \in \mathscr{K}(\Gamma)$.

6. The kernel of the compound game. The results of the preceding sections lead to the main theorem of this article, a theorem 
that determines the structure of the kernel of a compound game. This theorem, which is interesting in itself, enables shortcuts in the computations leading to the kernel of a decomposable game.

Theorem 6.1. Let $\Gamma=\Gamma_{0}\left[\Gamma_{1}, \cdots, \Gamma_{m}\right]$ be a monotonic dummyfree compound game with simple component games $\Gamma_{1}, \cdots, \Gamma_{m}$. Assume that every component that consists of more than one player is in 1-0-normalization ${ }^{4}\left(N_{k} \in \mathscr{W}^{k}\right.$ and for $\left.i \in N_{k}\{i\} \notin \mathscr{W}^{k}\right)$. Let $w_{k}$ denote the number of veto players in $\Gamma_{k}$ and $w=\left(w_{1}, \cdots, w_{m}\right)$. Under these conditions $x \in \mathscr{X}(\Gamma)$ belongs to the kernel, $\mathscr{K}(\Gamma)$, if and only if for every $k, k=1, \cdots, m$, such that $x\left(N_{k}\right)>0 B_{N_{k}} x \in \mathscr{K}\left(\Gamma_{k}\right)$ and the weak imputation $\mu[x]$ belongs to the weak w-equalizing set, $\widetilde{\mathscr{P}}^{w}\left(\Gamma_{0}\right)$, of the quotient game $\Gamma_{0}$.

Proof. (a) Suppose $x \in \mathscr{C}(\Gamma)$. Lemma 3.7 assures that the conditions of Theorems 4.1-4.2 are satisfied. From these theorems it follows that for every $k$ such that $x\left(N_{k}\right)>0 B_{N_{k}} x \in \mathscr{K}\left(\Gamma_{k}\right)$. Since for every $x \in \mathscr{X}(\Gamma)$

$$
x=\sum_{k=1}^{m} x\left(N_{k}\right) \cdot\left(B_{N_{k}} x\right)^{*}
$$

(for the *-notation refer to Lemma 5.6; if $x\left(N_{k}\right)=0$ for a certain $k$ we define $B_{N_{k}} x$ to be any point in $\mathscr{X}\left(\Gamma_{k}\right)$-anyhow it is multiplied by zero) it follows from Lemma 5.6 that $\mu[x] \in \widetilde{\mathscr{P}}^{w}\left(\Gamma_{0}\right)$.

(b) Suppose $x \in \mathscr{X}(\Gamma)$ is an imputation satisfying our conditions. Lemma 5.6 implies that $x \in \mathscr{C}(\Gamma)$.

CoRollary 6.2. Under the conditions of Theorem 6.1

$$
\begin{gathered}
\mathscr{K}(\Gamma)=\mathscr{X}(\Gamma) \cap\left\{\sum_{k=1}^{m} \frac{\hat{\mu}_{k}}{\mu_{k}\left[x^{k^{*}}\right]} x^{k^{*}}: \mu \in \widetilde{\mathscr{P}}^{w}\left(\Gamma_{0}\right),\right. \\
\left.x^{j} \in \mathscr{C}\left(\Gamma_{j}\right), 1 \leqq j \leqq m\right\} .
\end{gathered}
$$

Proof. Suppose $x \in \mathscr{K}(\Gamma)$. For every $k \in M$ such that $x\left(N_{k}\right)>0$ let $x^{k}=B_{N_{k}} x$ and let $\hat{\mu}=\mu[x]$. For $k \in M$ such that $x\left(N_{k}\right)=0$ let $x^{k}$ be any point in the kernel $\mathscr{K}\left(\Gamma_{k}\right)$. According to Theorem 6.1 $x^{k} \in \mathscr{K}\left(\Gamma_{k}\right)$ for every $k \in M$ and $\hat{\mu} \in \widetilde{\mathscr{P}}^{w}\left(\Gamma_{0}\right)$. The minimum payoff to a winning coalition is positive for every point in the kernel of a simple game (see [6; Lemma 3.7]). Thus, $\mu_{k}\left[x^{k^{*}}\right]>0$ and

$$
\frac{\mu_{k}}{\mu_{k}\left[x^{k^{*}}\right]}=x\left(N_{k}\right) \text {. }
$$

${ }^{4}$ The normalization assumption may be dropped and the theorem is true for the pseudo-kernel instead of the kernel (see Lemma 5.6). 
According to $(6.1)$

$$
x=\sum_{k=1}^{m} \frac{\widehat{\mu}_{k}}{\mu_{k}\left[x^{k^{*}}\right]} x^{k^{*}}
$$

and that proves that $\mathscr{K}(\Gamma)$ is obtained in the right-hand side of (6.2). Let $x$ belong to the right-hand side of (6.2). Thus, $x \in \mathscr{X}(\Gamma)$ and there exist $x^{k} \in \mathscr{K}\left(\Gamma_{k}\right), k=1, \cdots, m$, and $\widehat{\mu} \in \widetilde{\mathscr{P}}^{w}\left(\Gamma_{0}\right)$ such that (6.4) is satisfied. Necessarily, for every $k$ such that $x\left(N_{k}\right)>0 x^{k}=$ $B_{N_{k}} x$ and for all the $k \in M$

$$
\begin{aligned}
\mu_{k}[x] & =\min \left\{x(S): S \in \mathscr{W}^{k}\right\}=\min \left\{\frac{\widehat{\mu}_{k}}{\mu_{k}\left[x^{k^{*}}\right]} x^{k}(S): S \in \mathscr{W}^{k}\right\} \\
& =\frac{\widehat{\mu}_{k}}{\mu_{k}\left[x^{k^{*}}\right]} \mu_{k}\left[x^{k^{*}}\right]=\widehat{\mu}_{k}
\end{aligned}
$$

and hence $\mu[x] \in \widetilde{\mathscr{P}}^{w}\left(\Gamma_{0}\right)$. Theorem 6.1 implies $x \in \mathscr{K}^{-}(\Gamma)$.

Corollary 6.2 shows how the kernel of the compound game is obtained from the kernels of the components and the weak $w$-equalizing set for the quotient game. The next theorem shows how the kernel is obtained if we are restricted to vertices of certain polyhedra generating the components' kernels and to the vertices of the weak $w$-equalizing set.

THEOREM 6.3. Assume the conditions of Theorem 6.1. Let $\mathscr{K}\left(\Gamma_{k}\right)=\bigcup_{j=1}^{s_{k}} K_{j}^{k}, k=1, \cdots, m$, where $K_{j}^{k}, j=1, \cdots, s_{k}, k=1, \cdots, m$, are convex polyhedra in which $\mu_{k}[x]$ is a linear function of $x$ (see Lemma 3.1). Let $\widetilde{\mathscr{P}}^{w}\left(\Gamma_{0}\right)=\bigcup_{j=1}^{s_{0}} K_{j}^{0}$ where $K_{j}^{0}, j=1, \cdots, s_{0}$, are convex polyhedra. Under these conditions

$$
\begin{aligned}
\mathscr{K}(\Gamma)=\mathscr{X}(\Gamma) \cap \bigcup_{j_{0}=1}^{s_{0}} \cdots \bigcup_{j_{m}=1}^{s_{m}} \operatorname{conv}\left\{\sum_{k=1}^{m} \frac{\widehat{\mu}_{k}}{\mu_{k}\left[x^{k^{*}}\right]} x^{k^{*}}:\right. \\
\left.\hat{\mu} \in \operatorname{vert} K_{j_{0}}^{0}, x^{i} \in \operatorname{vert} K_{j_{i}}^{i}, i=1, \cdots, m\right\} .
\end{aligned}
$$

Proof. Define a mapping $\Psi: \widetilde{\mathscr{P}}^{w}\left(\Gamma_{0}\right) \times \mathscr{K}\left(\Gamma_{1}\right) \times \cdots \times \mathscr{\mathscr { C }}\left(\Gamma_{m}\right) \rightarrow E^{n}$ by

$$
\Psi\left(\widehat{\mu}, x^{1}, \cdots, x^{m}\right)=\sum_{k=1}^{m} \frac{\widehat{\mu}_{k}}{\mu_{k}\left[x^{k^{*}}\right]} x^{k^{*}} .
$$

According to Corollary 6.2

$$
\mathscr{K}(\Gamma)=\mathscr{X}(\Gamma) \cap \Psi\left[\widetilde{P}^{w}\left(\Gamma_{0}\right) \times \mathscr{K}\left(\Gamma_{1}\right) \times \cdots \times \mathscr{K}\left(\Gamma_{m}\right)\right] .
$$

If $1 \leqq j_{k} \leqq s_{k}, k=0,1, \cdots, m$, then the restriction of $\Psi$ to the set $K_{j_{0}}^{0} \times K_{j_{1}}^{1} \times \cdots \times K_{j_{m}}^{m}$ is defined everywhere and it is a multi-projective 
transformation since $\mu_{k}\left[x^{k^{*}}\right]$ is linear in $K_{j_{k}}^{k}, k=1, \cdots, m$. Thus, $\Psi$ is convexity-preserving in this domain (see (2.25)) and therefore,

$$
\begin{aligned}
\Psi\left[K_{j_{0}}^{0} \times \cdots \times K_{j_{m}}^{m}\right]= & \operatorname{conv} \Psi\left[\operatorname{vert} K_{j_{0}}^{0} \times \cdots \times \operatorname{vert} K_{j_{m}}^{m}\right] \\
= & \operatorname{conv}\left\{\Psi\left(\hat{\mu}, x^{1}, \cdots, x^{m}\right): \hat{\mu} \in \operatorname{vert} K_{j_{0}}^{0},\right. \\
& \left.x^{i} \in \operatorname{vert} K_{j_{i}}^{i}, i=1, \cdots, m\right\} .
\end{aligned}
$$

To complete the proof of the present theorem, notice that

$$
\begin{aligned}
\Psi\left[\widetilde{\mathscr{P}}^{w}\left(\Gamma_{0}\right)\right. & \left.\times \mathscr{K}\left(\Gamma_{1}\right) \times \cdots \times \mathscr{K}\left(\Gamma_{m}\right)\right] \\
& =\bigcup_{j_{0}=1}^{s_{0}} \cdots \bigcup_{j_{m}=1}^{s_{m}} \Psi\left[K_{j_{0}}^{0} \times \cdots \times K_{j_{m}}^{m}\right] .
\end{aligned}
$$

In case $\Gamma_{0}$ is a simple game without veto players the kernel of the compound game can be presented using $\mathscr{P}^{w}\left(\Gamma_{0}\right)$ instead of $\widetilde{\mathscr{P}}^{w}\left(\Gamma_{0}\right)$. This will be done by an appropriate modification in the definition of the mapping $\Psi$. Moreover, in this case the intersction with $\mathscr{Z}(\Gamma)$ can be omitted.

THEOREM 6.4. Under the conditions of Theorem 6.1, assume that $\Gamma_{0}$ is a simple game without veto players. Let $K_{j}^{k}, j=1, \cdots, s_{k}$, $k=1, \cdots, m$, be as in Theorem 6.3. Let $\mathscr{P}^{w}\left(\Gamma_{0}\right)=\bigcup_{j=1}^{s_{0}} K_{j}^{0}$ where $K_{j}^{0}$, $j=1, \cdots, s_{0}$, are convex polyhedra. Under these conditions

$$
\begin{aligned}
& \mathscr{\mathscr { C }}(\Gamma)=\bigcup_{J_{0}=1}^{s_{0}} \cdots \bigcup_{j_{m}=1}^{s_{m}} \operatorname{conv}\left\{\sum_{k=1}^{m} \frac{\hat{\mu}_{k} / \mu_{k}\left[x^{k^{*}}\right]}{\sum_{i=1}^{m} \hat{\mu}_{i} / \mu_{i}\left[2^{i^{*}}\right]} x^{k^{*}}:\right. \\
& \left.\hat{\mu} \in \operatorname{vert} K_{\jmath_{\omega}}^{0}, x^{i} \in \operatorname{vert} K_{j_{2}}^{i}, 1 \leqq i \leqq m\right\} .
\end{aligned}
$$

Proof. Since $\Gamma_{0}$ is a simple game without veto players, it follows from Lemma 5.4 that $\hat{\mu} \in \widetilde{\mathscr{P}}^{w}\left(\Gamma_{0}\right)$ if and only if $\hat{\mu} / \hat{\mu}(M) \in \mathscr{P}^{w}\left(\Gamma_{0}\right)$. It follows that all the vertices of $\widetilde{\mathscr{T}}^{w}\left(\Gamma_{0}\right)$ except the origin are vertices of $\mathscr{P}^{w}\left(\Gamma_{0}\right)$. Anyhow, the origin contributes nothing to (6.6) so that it can be omitted from vert $K_{j_{0}}^{0}$ (see (6.6)) and we may write $\mathscr{P}^{w}\left(\Gamma_{0}\right)$ instead of $\widetilde{\mathscr{T}}^{w}\left(\Gamma_{0}\right)$. Moreover, instead of intersecting with $\mathscr{X}(\Gamma)$ in the right-hand side of (6.6), we can obtain exact imputations by normalization, i.e., by defining

$$
\Psi\left(\hat{\mu}, x^{1}, \cdots, x^{m}\right)=\sum_{k=1}^{m} \frac{\hat{\mu}_{k} / \mu_{k}\left[2^{k *}\right]}{\sum_{j=1}^{m} \hat{\mu}_{j} / \mu_{j}\left[x^{j *}\right]} 2^{k^{*}} .
$$

REMARK 6.5. If $1, \cdots, l$ are the veto players in $\Gamma_{0}$ then either

$$
\Gamma=\Gamma_{1} \otimes \cdots \otimes \Gamma_{m}
$$


(in case $l=m$ ), or

$$
\Gamma=\Gamma_{l} \otimes \cdots \otimes \Gamma_{l} \otimes \Gamma_{0}^{\prime}\left[\Gamma_{l+1}, \cdots, \Gamma_{m}\right]
$$

(in case $1 \leqq l<m$ ) where $\Gamma_{0}^{\prime}$ is a monotonic simple game without veto players. The kernel of $\Gamma_{0}^{\prime}\left[\Gamma_{l+1}, \cdots, \Gamma_{m}\right]$ can be computed according to Theorem 6.4. Given the kernels of the components, the kernel of the product is very easy to compute (see [6; Theorem 3.1]). The set of vertices of a polyhedron in the kernel of a product is the union of sets of vertices of polyhedra in the kernels of the components.

ExAmple 6.6. Let $\Gamma_{6}$ be a monotonic simple game in which all the 3-player coalitions except $\{1,2,3\}$ and $\{4,5,6\}$ win. It is left to the reader to verify that the kernel of $\Gamma_{6}$ is the line segment $[(1 / 3$, $1 / 3,1 / 3,0,0,0),(0,0,0,1 / 3,1 / 3,1 / 3)]$. Denote

$$
x^{\alpha}=\left(\frac{\alpha}{3}, \frac{\alpha}{3}, \frac{\alpha}{3}, \frac{1}{3}-\frac{\alpha}{3}, \frac{1}{3}-\frac{\alpha}{3}, \frac{1}{3}-\frac{\alpha}{3}\right) .
$$

Thus,

$$
\mu\left(x^{\alpha}\right)=\min \left\{x^{\alpha}(S): S \in \mathscr{W}\right\}=\left\{\begin{array}{lll}
\frac{\alpha-1}{3} & \text { if } & 0 \leqq \alpha \leqq \frac{1}{2} \\
\frac{2-\alpha}{2} & \text { if } & \frac{1}{2} \leqq \alpha \leqq 1
\end{array} .\right.
$$

$\mu(x)$ is a linear function of $x$ in $K_{1} \equiv[(1 / 3,1 / 3,1 / 3,0,0,0),(1 / 6,1 / 6$, $1 / 6,1 / 6,1 / 6,1 / 6)]$ and in $K_{2} \equiv[(0,0,0,1 / 3,1 / 3,1 / 3),(1 / 6,1 / 6,1 / 6,1 / 6$, $1 / 6,1 / 6)]$. Consider the kernel of the game $\Gamma_{12}=\Gamma_{6} \oplus \Gamma_{6}$. The quotient game is $(\{1,2\} ;\{1\},\{2\},\{1,2\})$. There are no veto players in $\Gamma_{6}$. The $(0,0)$-equalizing set for the quotient game consists of a unique point - $(1 / 2,1 / 2)$. A vertex of $\mathscr{K}\left(\Gamma_{12}\right)$ is a combination of vertices of the polyhedra that generate $\mathscr{K}\left(\Gamma_{6}\right)$. The combination is determined by (6.12). For instance, if $x^{1}=(1 / 3,1 / 3,1 / 3,0,0,0)$ and $x^{2}=(1 / 6,1 / 6$, $1 / 6,1 / 6,1 / 6,1 / 6)$ then, since, necessarily, $\hat{\mu}=(1 / 2,1 / 2), x=\Psi\left(\hat{\mu}, x^{1}, x^{2}\right)=$ $(1 / 5,1 / 5,1 / 5,0,0,0,1 / 15,1 / 15,1 / 15,1 / 15,1 / 15,1 / 15)$. Because of the symmetry, each imputation $x$ in $\mathscr{K}\left(\Gamma_{12}\right)$ can be represented by a quadruple $\left(\alpha_{1} ; \alpha_{2} ; \alpha_{3} ; \alpha_{4}\right)$ where $\alpha_{1}=x_{1}=x_{2}=x_{3}, \alpha_{2}=x_{4}=x_{5}=x_{6}$ etc. The kernel of $\Gamma_{12}$ consists of the following four quadrangles, presented by their vertices. (a) $A E F O$ (b) $B E H O$ (c) $C G F O$ (d) $D G H O$, where $A=(1 / 6 ; 0 ; 1 / 6 ; 0), B=(1 / 6 ; 0 ; 0 ; 1 / 6), C=(0 ; 1 / 6 ; 1 / 6 ; 0), D=(0 ; 1 / 6$; $0 ; 1 / 6), \quad E=(1 / 5 ; 0 ; 1 / 15 ; 1 / 15), \quad F=(1 / 15 ; 1 / 15 ; 1 / 5 ; 0), \quad G=(0 ; 1 / 5$; $1 / 15 ; 1 / 15), H=(1 / 15 ; 1 / 15 ; 0 ; 1 / 5)$, and $O=(1 / 12 ; 1 / 12 ; 1 / 12 ; 1 / 12)$.

EXAMPLE 6.7. Let $\square$ be a 4-player monotonic simple game whose minimal winning coalitions are $\{1,3\},\{2,3\},\{1,4\},\{2,4\} . \mathscr{C}(\square)$ is the 


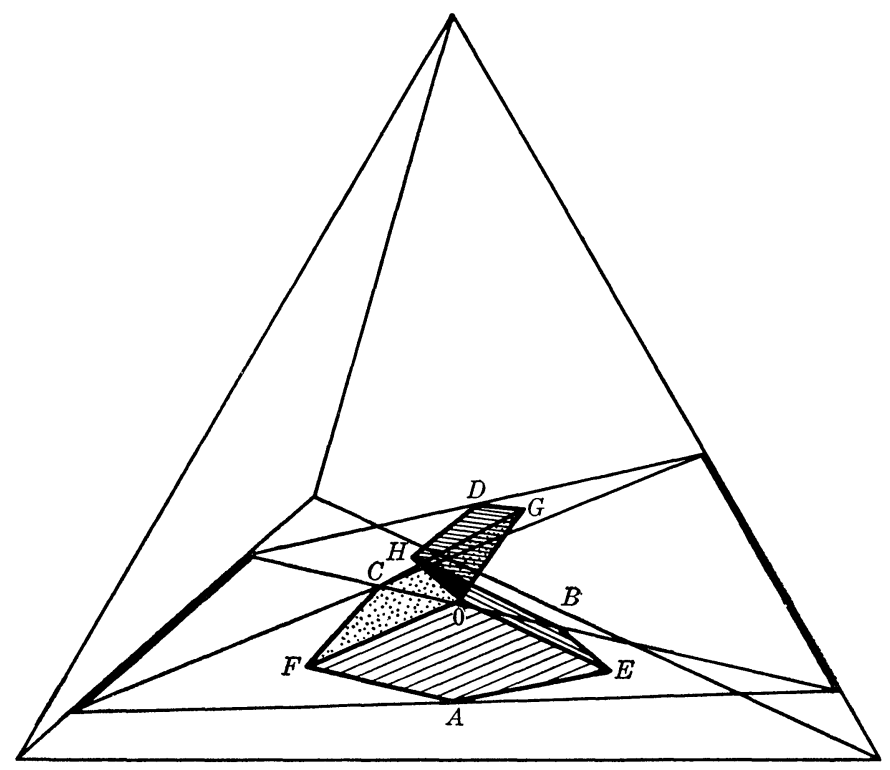

FIG. 6.1

line segment $[(1 / 2,1 / 2,0,0),(0,0,1 / 2,1 / 2)]$ (notice that $\square=B_{2}^{*} \otimes B_{2}^{*}$; see (2.11)). The function $\mu(x)$ is constant over $\mathscr{K}(\square)(\mu(x)=1 / 2)$. Consider the kernel of the 10-player game $\Gamma=M_{3}\left[\square, M_{3}, M_{3}\right]$ (see Example 5.5). $\mathscr{K}\left(M_{3}\right)$ consists of the unique point $(1 / 3,1 / 3,1 / 3)$. $\mathscr{P}^{(0,0,0)}\left(M_{3}\right)$ was shown to consist of three line segments having a

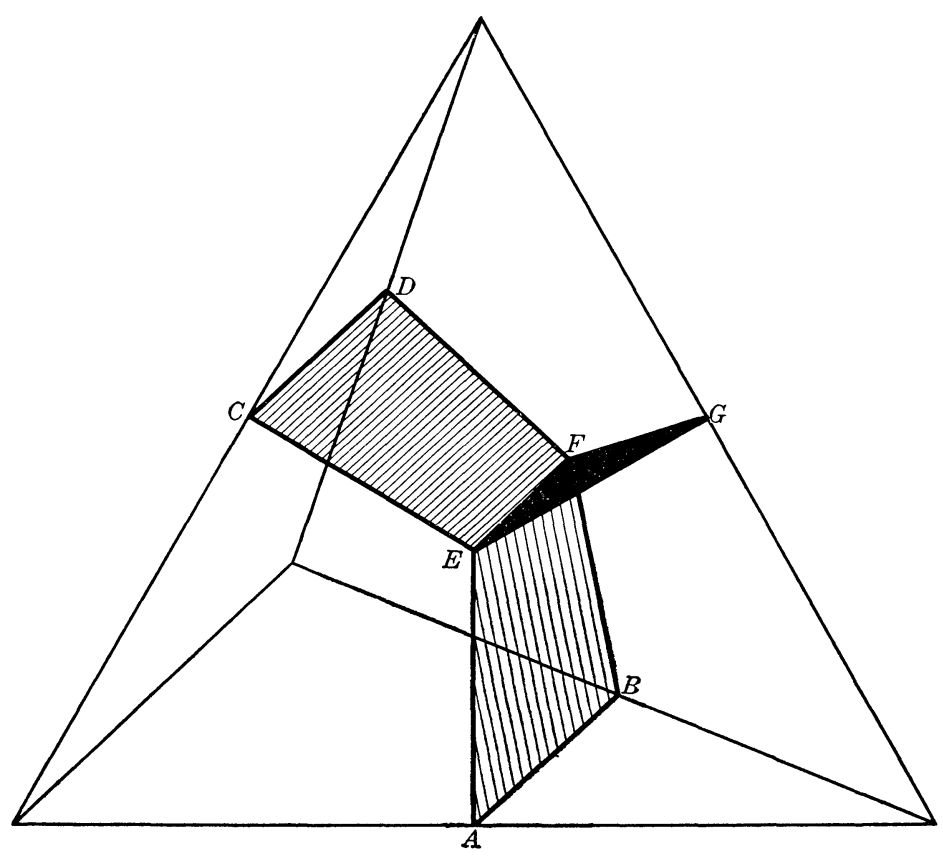

FIG. 6.2 
common vertex. Let $x^{1}=(1 / 2,1 / 2,0,0) \in \mathscr{K}(\square), x^{2}=x^{3}=(1 / 3,1 / 3$, $1 / 3)$ and $\hat{\mu}=(1 / 2,0,1 / 2) \in \mathscr{P}^{(0,0,0)}\left(M_{3}\right)$. It can be verified that $\Psi\left(\hat{\mu}, x^{1}\right.$, $\left.x^{2}, x^{3}\right)=(2 / 7,2 / 7,0,0,0,0,0,1 / 7,1 / 7,1 / 7)$. Running over all the possible combinations we find that $\mathscr{K}(\Gamma)$ consists of the two quadrangles (a) $A B E F$ (b) $C D E F$ and the triangle (c) $G E F$, where $A=(2 / 7 ; 0 ; 1 / 7 ; 0)$, $B=(0 ; 2 / 7 ; 1 / 7 ; 0), C=2 / 7 ; 0 ; 0 ; 1 / 7), D=(0 ; 2 / 7 ; 0 ; 1 / 7), E=(1 / 5 ; 0$; $1 / 10 ; 1 / 10), F=(0 ; 1 / 5 ; 1 / 10 ; 1 / 10)$ and $G=(0 ; 0 ; 1 / 6 ; 1 / 6)$.

7. Kernels of compound majority games. A majority game is an $n$-player simple game $M_{n, k}$ in which a coalition wins if and only if it consists of at least $k$ players. In this section we apply the results of the preceding one to games of the form

$$
\Gamma=M_{n_{0}, k_{0}}\left[M_{n_{1}, k_{1}}, \cdots, M_{n_{m}, k_{m}}\right]
$$

where $m=n_{0}$ and $0<k_{i}<n_{i}, i=0,1, \cdots, m$.

LEMMA 7.1. Let $x \in \mathscr{X}\left(M_{n, k}\right)$ and denote

$$
\mathscr{D}=\{S:(\forall T \subset N)(e(S, x) \geqq e(T, x))\} \text {. }
$$

Under these conditions if $S, T \in \mathscr{D}$ and $i, j \in(S \cup T) \backslash(S \cap T)$ then $x_{i}=x_{j}$.

Proof. Assume $i \in S \backslash T$ and $j \in T \backslash S$. Thus,

$$
e(S, x)=e[(S \backslash\{i\}) \cup\{j\}, x]
$$

and therefore

$$
x_{i} \leqq x_{j}
$$

Similarly,

$$
x_{j} \leqq x_{i}
$$

If $i, j \in S \backslash T$ let $l \in T \backslash S$ (if $S \supset T$ then, clearly, $x_{i}=x_{j}=0$ ) and according to what we have proved in (7.4) and (7.5) $x_{i}=x_{l}=x_{j}$.

Lemma 7.2. Let $x$ and $\mathscr{D}$ be as in Lemma 7.1. If $S_{1}, \cdots, S_{r} \in \mathscr{D}$ and $i, j \in \mathbf{U}_{p=1}^{r} S_{p} \backslash \bigcap_{p=1}^{r} S_{p}$ then $x_{i}=x_{j}$.

Proof. (a) Assume that there is $p, 1 \leqq p \leqq r$, such that $i, j \notin S_{p}$. If there is $q$ such that $i, j \in S_{q}$ then $i, j \in\left(S_{p} \cup S_{q}\right) \mid\left(S_{p} \cap S_{q}\right)$ and we can apply Lemma 7.1. If there is no such $q$ let $s$ be such that $i \in S_{s}$ and let $t$ be such that $j \in S_{t}$ and Lemma 7.1 can be applied again.

(b) Suppose that for every $p, p=1, \cdots, r$, either $i \in S_{p}$ or $j \in S_{p}$. Let $p$ be such that $i \notin S_{p}$ (and therefore $j \in S_{p}$ ) and let $q$ be such that 
$j \notin S_{q}$. Thus, $i, j \in\left(S_{p} \cup S_{q}\right) \backslash\left(S_{p} \cap S_{q}\right)$ and Lemma 7.1 can be applied.

Lemma 7.3. If $i \in \mathbf{U}_{p=1}^{r} S_{p} \backslash \bigcap_{p=1}^{r} S_{p}$ and $j \in \bigcap_{p=1}^{r} S_{p}$ where $x, \mathscr{D}$, $S_{1}, \cdots, S_{r}$ are as in the preceding Lemma, then $x_{i} \geqq x_{j}$.

Proof. Let $T \in \mathscr{D}$ be such that $i \notin T$ (clearly, $j \in T$ ) and apply (7.5).

REMARK 7.4. An imputation $x \in \mathscr{X}\left(M_{n, k}\right)$ belongs to $\mathscr{P}^{(0, \ldots, 0)}\left(M_{n, k}\right)$ if and only if $\bigcup_{S \in \mathscr{D}} S=N$, where $\mathscr{D}$ is defined by (7.2) (see Definition 5.1 condition (i)).

THEOREM 7.5. Let $x \in \mathscr{X}\left(M_{n, k}\right) . \quad x \in \mathscr{P}^{0}\left(M_{n, k}\right)$ if and only if there is $S \subset N$ such that $|S|=k-1$ and for every $l \in S$ and $i, j \notin S$ $x_{i}=x_{j} \geqq x_{l}$.

Proof. (a) Assume that there is a coalition $S$ as specified in the theorem. In this case all the $k$-player coalitions $T$ containing $S$ have the same payoff. Thus, this collection of coalitions is exactly $\mathscr{D}$ and since it covers $N$ it follows that $x \in \mathscr{P}^{0}\left(M_{n, k}\right)$ (Remark 7.4).

(b) Assume, conversely, that $x \in \mathscr{P}^{0}\left(M_{n, k}\right)$. Let $\widetilde{S}=\bigcap_{S \in \Xi} S$. According to Lemmas 7.2-7.3 and Remark 7.4, for every $l \in \widetilde{S}$ and $i, j \notin \widetilde{S} x_{i}=x_{j} \geqq x_{l}$. The maximum excess is achieved in a $k$-player coalition. Since $S$ is an intersection of a collection of $k$-player coalitions covering $N(k<n)$ it follows that $|\widetilde{S}| \leqq k-1$. Obviously, every $(k-1)$-player coalition containing $\widetilde{S}$ satisfies the condition concerning $l, i$, and $j$.

CoRollary 7.6. Denote by $a^{S}(S \subset N)$ an imputation such that $a_{i}^{S}=1 /|S|$ for $i \in S$ and $a_{i}^{S}=0$ for $i \notin S$. Then

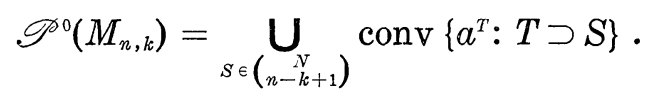

Proof. Let $S \in\left(\begin{array}{c}N \\ n-k+1\end{array}\right)$ and denote by $\mathscr{A}_{S}$ the set of all the imputations $x$ such that for every $l \notin S$ and $i, j \in S x_{i}=x_{j} \geqq x_{l}$.

(a) Let $\lambda_{T} \geqq 0, T \supset S$ be such that $\sum_{T \supset S} \lambda_{T}=1$. Let $x=\sum_{T \supset S} \lambda_{T} a^{T}$. Then

$$
x= \begin{cases}\sum_{T \rightarrow S} \frac{\lambda_{T}}{|T|} & \text { if } \quad i \notin S \\ \sum_{T \supset S} \frac{\lambda_{T}}{|T|} & \text { if } \quad i \in S\end{cases}
$$

and therefore $x \in \mathscr{A}_{S}$. We have, thus, proved that $\mathscr{A}_{S} \supset \operatorname{conv}\left\{a^{T}\right.$ : 
$T \supset S\}$

(b) Let $x \in \mathscr{A}_{S}$. Without loss of generality assume that $S=$ $\{k, k+1, \cdots, n\}$ and the players are arranged so that $x_{1} \leqq x_{2} \leqq \cdots \leqq$ $x_{k-1} \leqq x_{k}=\cdots=x_{n}$. Since $\sum_{i=1}^{n} x_{i}=1$ it follows that $x_{1} \leqq 1 / n$ and for every $i$

$$
x_{i} \leqq \frac{1-\sum_{j=1}^{i-1} x_{j}}{n-i+1}
$$

Let $T_{1}=N$ and for every $i, i=1, \cdots, k-1, T_{i+1}=T_{i} \backslash\{i\}$. Let $\alpha_{1}=n x_{1}$ and for every $i, i=2, \cdots, k-1, \alpha_{i}=(n-i+1) \cdot\left(x_{i}-x_{i-1}\right)$. Then $\alpha_{i} \geqq 0, i=1, \cdots, k-1$, and

$$
\begin{aligned}
\sum_{i=1}^{n-1} \alpha_{i} & =n x_{1}+\sum_{i=2}^{n-1}(n-i+1) \cdot\left(x_{i}-x_{i-1}\right) \\
& =(n-k+2) \cdot x_{k+1}+\sum_{i=1}^{k-2} x_{i}=1-\sum_{k=i}^{k-2} x_{i}+\sum_{i=1}^{k-2} x_{i}=1 .
\end{aligned}
$$

Define $\alpha_{k}=1-\sum_{i=1}^{k-1} \alpha_{i}$ and $y=\sum_{i=1}^{k} \alpha_{i} \alpha^{T_{i}}$. Also, for every $j, j=1$, $\cdots, n$, let $j^{*}=\min (j, k)$. Then

$$
y_{j}=\sum_{i=1}^{j^{*}} \alpha_{i} \cdot \frac{1}{n-i+1}=\sum_{i=2}^{j^{*}}\left(x_{i}-x_{i-1}\right)-x_{1}=x_{j^{\star}}=x_{j} .
$$

Hence, $\mathrm{y}=x$. We have proved that $x \in \operatorname{conv}\left\{a^{T_{i}}: i=1, \cdots, k\right\} \subset$ conv $\left\{a^{T}: T \supset S\right\}$. Thus, $\mathscr{A}_{S}=\operatorname{conv}\left\{a^{T}: T \supset S\right\}$. According to Theorem 7.5 $\mathscr{P}^{0}\left(M_{n, k}\right)=\bigcup_{S \in\left(\begin{array}{c}N \\ n-k+1\end{array}\right)} \mathscr{A}_{S}(|N| S \mid=k-1)$ and this completes the proof.

Let $\Gamma$ be the game defined in (7.1). For every $S \subset M(S \neq \varnothing)$ denote by $b^{S}$ an imputation in $\Gamma$ such that for every $i \in N_{l}(l=1, \cdots, m)$

$$
b_{i}^{S}=1 /\left(k_{l} \cdot \sum_{j \in S} \frac{n_{j}}{k_{j}}\right) \text { if } l \in S \text { and } b_{i}^{S}=0 \text { if } l \notin S .
$$

Theorem 7.7. Let $\Gamma$ be the game defined in (7.1). Then

$$
\mathscr{K}(\Gamma)=\underset{S \in\left(\begin{array}{c}
M \\
m-k_{0}+1
\end{array}\right)}{\bigcup} \operatorname{conv}\left\{b^{T}: T \supset S\right\} .
$$

Proof. Because of the symmetry, $\mathscr{K}\left(M_{n, k}\right)$ consists of a unique point $-(1 / n, \cdots, 1 / n)$. The minimum payoff to a winning coalition is therefore $k / n$. According to Corollary $7.8 \mathscr{P}^{0}\left(M_{n_{0}, k_{0}}\right)$ is the union of the polyhedra $\mathscr{A}_{S}\left(S \subset M,|S|=m-k_{0}-1\right)$ whose vertices are $a^{T}, T \supset S$. The combination of the components' kernels defined by $a^{T}, T \subset M$, (see (6.12)) is the imputation $x \in \mathscr{X}(\Gamma)$ where for every $i \in N_{l}(l=1, \cdots, m)$ 


$$
\begin{aligned}
x_{i} & =\frac{a_{l}^{T} /\left(k_{l} / n_{l}\right)}{\sum_{j=1}^{m} a_{j}^{T} /\left(k_{j} / n_{j}\right)} \cdot \frac{1}{n_{l}}=\frac{1}{k_{l} \sum_{j \in T} a_{j}^{T} \cdot n_{j} / k_{j}} \cdot n_{l} a_{l}^{T} \cdot \frac{1}{n_{l}} \\
& =\left\{\begin{array}{ccc}
1 /\left(k_{l} \sum_{j \in T} \frac{n_{l}}{k_{j}}\right) & \text { if } & l \notin T \\
0 & \text { if } & l \notin T
\end{array}\right\}=b_{i}^{T} .
\end{aligned}
$$

It follows from Theorem 6.4 that $\mathscr{K}(\Gamma)$ is the union of the polyhedra $Q_{S}\left(S \subset M,|S|=m-k_{0}+1\right)$ whose vertices are the $b^{T}$-s $(T \supset S)$.

Acknowledgment. The author is indebted to Professor Michael Maschler of the Hebrew University of Jerusalem for his helpful advices and suggestions.

\section{REFERENCES}

1. R. J. Aumann, B. Peleg, and D. Rabinowitz, A method for computing the kernel of n-person games, Mathematics of Computation, 19 (1965), 531-551.

2. M. Davis and M. Maschler, The kernel of a cooperative game, Naval Research Logistics Quarterly, 12 (1965), 223-259.

3. A. Kopelowitz, Computation of the kernels of simple games and of the nucleolus of $n$-person games, Research Program in Game Theory and Mathematical Economics, Research Memorandum No. 31, Department of Mathematics, The Hebrew University of Jerusalem, Jerusalem, 1967.

4. M. Maschler and B. Peleg, A characterization, existence proof and dimension bounds for the kernel of a game, Pacific J. Math., 18 (1966), 289-328.

5 . - The structure of the kernel of a cooperative game, SIAM J. Appl. Math., 15 (1967), 569-604.

6. N. Megiddo, The kernel and the nucleolus of a product of simple games, Israel J. Math., 9 (1971), 210-221.

7. - Tensor decomposition of cooperative games, Research Program in Game Theory and Mathematical Economics, Research Memorandum No. 71, Department of Mathematics, The Hebrew University of Jerusalem, Jerusalem, 1972. (To appear in SIAM J. Appl. Math.)

8. - Nucleoluses of compound simple games, SIAM J. Appl. Math., 26 (1974).

9. G. Owen, The Tensor Composition of Nonnegative Games, Advances in Game Theory, M. Dresher, L. S. Shapley, and A. W. Tucker, eds., Annals of Mathematics Studies, No.

52, Princeton University Press, Princeton, (1964), 307-327.

10. B. Peleg, The kernel of the composition of characteristic function games, Israel J. Math., 3 (1965), 127-138.

11. L. S. Shapley, Simple Games: An Outline of the Descriptive Theory, Behavioral Science, 7 (1962), 59-66 (also The RAND Corporation, P-2277, April, 1961).

12. - Compound Simple Games I: Solutions of Sums and Products, The RAND Corporation, RM-3192, June, 1962.

13. - Compound Simple Games II: Some General Composition Theorems, The RAND Corporation, RM-3643, July, 1963.

14. Solu tions of Compound Simple Games, Advances in Game Theory, M. Dresher,

L. S. Shapley, and A. W. Tucker, eds., Annals of Mathematics Studies, No. 52, Princeton University Press, Princeton, (1964), 267-305. 
15.

Compound Simple Games III: On Committees, The RAND Corporation, RM-5438-PR, October, 1967 (also in "New Methods of Thought and Procedure", ed. by F. Zwycky and A. Wilson, Springer, New York, 1968).

Received September 28, 1972 and in revised form March 20, 1973.

Tel Aviv University 



\section{PACIFIC JOURNAL OF MATHEMATICS}

\section{EDITORS}

RICHARD ARENS (Managing Editor)

University of California

Los Angeles, California 90024

\section{R. A. Beaumont \\ University of Washington \\ Seattle, Washington 98105}

\section{J. DugundjI*}

Department of Mathematics University of Southern California Los Angeles, California 90007

D. Gilbarg and J. Milgram

Stanford University

Stanford, California 94305

\section{ASSOCIATE EDITORS}
E. F. BeCKenBaCH
B. H. NeumanN
F. WOLF
K. YoSHIDA

\section{SUPPORTING INSTITUTIONS}

\author{
UNIVERSITY OF BRITISH COLUMBIA \\ CALIFORNIA INSTITUTE OF TECHNOLOGY \\ UNIVERSITY OF CALIFORNIA \\ MONTANA STATE UNIVERSITY \\ UNIVERSITY OF NEVADA \\ NEW MEXICO STATE UNIVERSITY \\ OREGON STATE UNIVERSITY \\ UNIVERSITY OF OREGON \\ OSAKA UNIVERSITY
}

\author{
UNIVERSITY OF SOUTHERN CALIFORNIA \\ STANFORD UNIVERSITY \\ UNIVERSITY OF TOKYO \\ UNIVERSITY OF UTAH \\ WASHINGTON STATE UNIVERSITY \\ UNIVERSITY OF WASHINGTON

$* * *$
$*$
AMERICAN MATHEMATICAL SOCIETY
NAVAL WEAPONS CENTER

The Supporting Institutions listed above contribute to the cost of publication of this Journal, but they are not owners or publishers and have no responsibility for its content or policies.

Mathematical papers intended for publication in the Pacific Journal of Mathematics should be in typed form or offset-reproduced, (not dittoed), double spaced with large margins. Underline Greek letters in red, German in green, and script in blue. The first paragraph or two must be capable of being used separately as a synopsis of the entire paper. Items of the bibliography should not be cited there unless absolutely necessary, in which case they must be identified by author and Journal, rather than by item number. Manuscripts, in duplicate if possible, may be sent to any one of the four editors. Please classify according to the scheme of Math. Rev. Index to Vol. 39. All other communications to the editors should be addressed to the managing editor, or Elaine Barth, University of California, Los Angeles, California, 90024.

100 reprints are provided free for each article, only if page charges have been substantially paid. Additional copies may be obtained at cost in multiples of 50 .

The Pacific Journal of Mathematics is issued monthly as of January 1966. Regular subscription rate: $\$ 60.00$ a year (6 Vols., 12 issues). Special rate: $\$ 30.00$ a year to individual members of supporting institutions.

Subscriptions, orders for back numbers, and changes of address should be sent to Pacific Journal of Mathematics, 103 Highland Boulevard, Berkeley, California, 94708.

PUBLISHED BY PACIFIC JOURNAL OF MATHEMATICS, A NON-PROFIT CORPORATION

Printed at Kokusai Bunken Insatsusha (International Academic Printing Co., Ltd.), 270, 3-chome Totsuka-cho, Shinjuku-ku, Tokyo 160, Japan

* C. R. DePrima California Institute of Technology, Pasadena, CA 91109, will replace J. Dugundji until August 1974. 


\section{Pacific Journal of Mathematics}

Vol. 50, No. $2 \quad$ October, 1974

Mustafa Agah Akcoglu, John Philip Huneke and Hermann Rost, A counter example to the Blum Hanson theorem in general spaces .............

Huzihiro Araki, Some properties of modular conjugation operator of von

Neumann algebras and a non-commutative Radon-Nikodym theorem

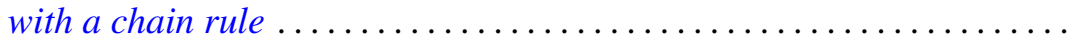

E. F. Beckenbach, Fook H. Eng and Richard Edward Tafel, Global properties of rational and logarithmico-rational minimal surfaces .....

David W. Boyd, A new class of infinite sphere packings ............. 383

K. G. Choo, Whitehead Groups of twisted free associative algebras ........

Charles Kam-Tai Chui and Milton N. Parnes, Limit sets of power series

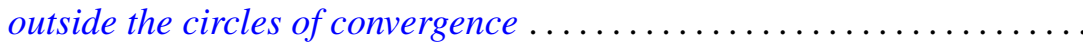

Allan Clark and John Harwood Ewing, The realization of polynomial

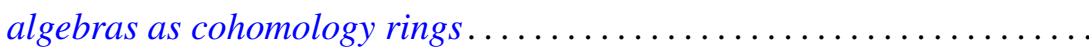

Dennis Garbanati, Classes of circulants over the p-adic and rational

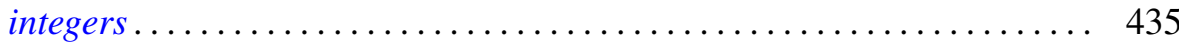

Arjun K. Gupta, On a "square" functional equation ................... 449

David James Hallenbeck and Thomas Harold MacGregor, Subordination and extreme-point theory ............................. 455

Douglas Harris, The local compactness of $v X \ldots \ldots . . . . . . . . . . . .4469$

William Emery Haver, Monotone mappings of a two-disk onto itself which fix the disk's boundary can be canonically approximated by homeomorphisms .................................. 477

Norman Peter Herzberg, On a problem of Hurwitz .................. 485

Chin-Shui Hsu, A class of Abelian groups closed under direct limits and subgroups formation ............................... 495

Bjarni Jónsson and Thomas Paul Whaley, Congruence relations and multiplicity types of algebras.....................

Lowell Duane Loveland, Vertically countable spheres and their wild sets.

Nimrod Megiddo, Kernels of compound games with simple components ....

Russell L. Merris, An identity for matrix functions ........ . .

E. O. Milton, Fourier transforms of odd and even tempered distributions ...

Dix Hayes Pettey, One-one-mappings onto locally connected generalized continua

Mark Bernard Ramras, Orders with finite global dimension

Doron Ravdin, Various types of local homogeneity. .

George Michael Reed, On metrizability of complete Moore spaces ...

Charles Small, Normal bases for quadratic extensions ..

Philip C. Tonne, Polynomials and Hausdorff matrices.... . . 